\title{
The importance of the longest R-R interval on 24-hour electrocardiography for mortality prediction in patients with atrial fibrillation
}

\author{
Dorota Zyśko', Anders Persson², Marta Obremska ${ }^{3}$, Marcin Leśkiewicz', \\ Artur Fedorowski ${ }^{2,4}$, Richard Sutton ${ }^{2,5}$, Linda S. B. Johnson ${ }^{2}$ \\ 1 Department of Emergency Medicine, Wroclaw Medical University, Wrocław, Poland \\ 2 Department of Clinical Sciences, Lund University, Malmö, Sweden \\ 3 Department of Preclinical Research, Wroclaw Medical University, Wrocław, Poland \\ 4 Department of Cardiology, Skåne University Hospital, Malmö, Sweden \\ 5 National Heart and Lung Institute, Imperial College, Hammersmith Hospital, London, United Kingdom
}

\section{KEY WORDS}

ambulatory electrocardiography, asystole, atrial fibrillation, mortality, pacemaker
Correspondence to:

Marta Obremska, MD, PhD, Department of Preclinical Research, Wroclaw Medical University, ul. Bartla 5 , 50-996 Wrocław, Poland, phone: +48717841813 , email: marta.obremska@umed.wroc.pl Received: October 8, 2020. Revision accepted: February 5, 2021. Published online: February 17, 2021. Kardiol Pol. 2021; 79 (3): 311-318 doi:10.33963/KP.15820 Copyright by the Author(s), 2021

\section{ABSTRACT}

BACKGROUND Heart rate control in atrial fibrillation (AF) is typically assessed by 24-hour electrocardiography (ECG). There are scarce data on the use of 24-hour ECG parameters to predict mortality in patients with AF. AIMS We aimed to identify 24 -hour ECG parameters that predict mortality in patients with AF.

METHODS We enrolled 280 ambulatory patients (mean [SD] age, 72 [8.7] years; $57.9 \%$ men) with permanent or persistent AF. Data on mortality and pacemaker or defibrillator implantation during follow-up were collected. Predictors of mortality were assessed using the Cox proportional hazards model and C statistic. RESULTS Compared with survivors, 78 patients (28\%) who died were older, more often had comorbidities, left bundle branch block (LBBB), reduced left ventricular ejection fraction, lower maximum heart rate, higher number of ventricular extrasystoles, and the longest R-R interval below 2 seconds. Univariate analysis showed higher mortality in patients with the longest $R-R$ intervals below 2 seconds compared with those with R-R intervals of 2 seconds or longer $(P<0.001)$. Independent mortality predictors in the regression model included older age, renal failure, history of coronary intervention, chronic obstructive pulmonary disease, LBBB, and a high number $(\geq 770)$ or absence of R-R intervals of at least 2 seconds. The area under the curve for mortality prediction increased after including ECG parameters $(0.748 ; 95 \% \mathrm{CI}$, $0.686-0.81$; vs $0.688 ; 95 \% \mathrm{CI}, 0.618-0.758 ; P=0.02$ ).

CONCLUSIONS A high number of R-R intervals longer than 2 seconds or their absence on 24-hour ECG may predict mortality in patients with AF.

INTRODUCTION Atrial fibrillation (AF) is the most common sustained cardiac arrhythmia and is known to be an independent predictor of mortality. ${ }^{1-3}$ Moreover, patients receiving anticoagulation most often die of cardiovascular causes. ${ }^{4}$ It has been also shown that 24-hour ambulatory electrocardiography (ECG) can be used to predict $\mathrm{AF}^{5-8}$ and mortality. ${ }^{9}$ However, there are scarce data on the use of 24-hour ECG parameters, including heart rate variables, in the prediction of mortality in patients with permanent
AF. Although heart rate control is the cornerstone of AF management, ${ }^{10}$ the best parameter for the assessment of optimal rate control in patients with AF has not been identified so far. Furthermore, cardiovascular autonomic dysfunction, which is characterized by increased sympathetic activity, reduced heart rate variability, and orthostatic hypotension, ${ }^{11}$ is a known predictor of AF and all-cause mortality and may potentially be reflected by 24-hour ECG recording. ${ }^{12-14}$ Thus, parameters derived from 24-hour 


\section{WHAT'S NEW?}

Cardiovascular autonomic dysfunction, which is characterized by increased sympathetic activity, is a known predictor of all-cause mortality. In patients with atrial fibrillation (AF), an increase in sympathetic activity is known to affect the conduction of electrical impulses of the atria and to shorten the R-R intervals. Moreover, a high ventricular rate during AF can lead to tachycardia-induced cardiomyopathy and is associated with unfavorable prognosis. We showed that 24-hour electrocardiography parameters such as the duration and number of R-R intervals can predict mortality in patients with AF. Survival was adversely affected by the absence of $R-R$ intervals lasting at least 2 seconds as well as by a high number of such intervals. We propose that a moderate number of R-R intervals lasting as least 2 seconds in patients with AF on 24-hour electrocardiography indicates adequate parasympathetic control.

ECG might be used to identify a subgroup of patients with AF who could benefit from a more aggressive treatment and follow-up, including therapies targeting autonomic nervous system activity. Therefore, the aim of this study was to determine whether common 24-hour ECG parameters may predict mortality in patients with persistent or permanent AF.

METHODS Study population The study was retrospective and included a group of $280 \mathrm{pa}-$ tients with permanent or persistent AF treated at a cardiac outpatient clinic at 2 locations (Wrocław and Krotoszyn, Poland) between 2004 and 2017. Electrocardiography was performed using the standard 3-channel Holter monitor (Aspel, Zabierzów, Poland and Edan Instruments, Shenzhen, China). The mean heart rate was defined as the average heart rate during the 24-hour ECG recording. The minimum and maximum heart rates were defined as the lowest and highest average heart rates, respectively, during the 5 consecutive R-R intervals. The following 24-hour ECG parameters were obtained from outpatients clinics: mean, maximum, and minimum 24-hour heart rate, the number of ventricular extrasystoles (VESs), the longest R-R interval, and the number of R-R intervals lasting at least 2 seconds.

Demographic and clinical data were obtained from digital patient records. The data included age, sex, left ventricular ejection fraction (LVEF), left atrial size, comorbidities such as diabetes, hypertension, coronary artery disease, and chronic obstructive pulmonary disease (COPD), type of AF (permanent or paroxysmal/persistent), history of pacemaker or defibrillator implantation, and the use of medications ( $\beta$-blockers, nondihydropyridine calcium channel blockers, amiodarone, or digoxin). $\beta$-Blockers dosages were presented as metoprolol equivalent dosages as follows: metoprolol $100 \mathrm{mg}$ = bisoprolol $10 \mathrm{mg}$ = carvedilol $50 \mathrm{mg}$ = atenolol $100 \mathrm{mg}=$ nebivolol $10 \mathrm{mg}$ = sotalol $160 \mathrm{mg}=$ betaxolol $10 \mathrm{mg},{ }^{15}$ and were reported as median and interquartile ranges. Left ventricular ejection fraction was considered to be reduced if the value was lower than $50 \%$. Diabetes was defined as a self-reported diagnosis established by a physician, or the current use of antidiabetic agents, or a history of hyperglycemia (fasting plasma glucose $\geq 127 \mathrm{mg} / \mathrm{dl}$ on 2 occasions or an oral glucose tolerance test with a plasma glucose level $>200 \mathrm{mg} / \mathrm{dl}$ ). Hypertension was defined as a self-reported diagnosis established by a physician, a history of elevated blood pressure and the use of antihypertensive drugs, or resting blood pressure of 140/90 mm Hg or higher on 2 measurements 1 week apart. A history of ischemic heart disease was defined as previous myocardial infarction, percutaneous coronary intervention, or coronary artery bypass grafting or the presence of significant coronary artery stenosis ( $>50 \%)$. Valvular heart disease was defined as the presence of moderate or severe valvular disease or a history of surgery for valvular disease. Permanent AF was diagnosed when there has been a joint decision by the patient and clinician to cease further attempts to restore sinus rhythm and there have been no ECG recordings showing sinus rhythm in the previous year. The outcome was total mortality which was ascertained via linkage of personal identification numbers (PESEL) to the Universal Electronic System for Registration of Population.

Data on pacemaker or defibrillator implantation during the period after index 24-hour ECG and data regarding survival during follow-up were obtained from the databases of the Lower Silesia and Greater Poland divisions of the Polish National Health Fund.

The study population was stratified according to the duration of the R-R interval: less than 2 seconds or 2 seconds or longer. Furthermore, the distribution of pauses lasting 2 to $2.9 \mathrm{sec}-$ onds and 3 or more seconds was presented. Finally, the absolute number of intervals lasting 2 seconds or longer was calculated for each patient. The study was approved by a bioethical committee (number 39/2019).

Statistical analysis Statistical analysis was performed using the Statistica 13.3 software (TIBCO Software Inc., Palo Alto, California, United States). Categorical variables were presented as numbers and percentages and compared using the Pearson $\mathrm{X}^{2}$ test with Yates correction, when necessary. Continuous variables with normal distribution confirmed by the Shapiro-Wilk test were presented as means (SD), and compared with the $t$ test. Finally, continuous variables which were not normally distributed were presented as medians and interquartile ranges (IQRs) and compared with the Mann-Whitney test. Kaplan-Meier curves were generated to visualize associations between 
TABLE 1 Baseline characteristics of the whole study group as well as deceased patients and survivors at the end of follow-up

Parameter

Age, $y$, mean (SD)

Male sex

Follow-up, mo, median (IQR)

Atrial fibrillation type

Hypertension

Ischemic heart disease

PCI/CABG

Myocardial infarction

Diabetes

Valvular heart disease

COPD

Any cancer diagnosis

Renal failure grade 4-5

LVEF, \%, median (IQR)

LVEF $<50 \%$

Left atrial size, $\mathrm{cm}$, median (IQR)

History of pacemaker or defibrillator implantation

Pacemaker / defibrillator implantation during follow-up

Time to implantation during follow-up, mo, median (IQR)

\section{4-hour ECG parameters}

Mean heart rate, bpm, median (IQR)

Minimum heart rate, bpm, median (IQR)

Maximum heart rate, bpm, median (IQR)

LBBB

RBBB

VESs, n, median (IQR)

The number of VESs $>5$

The longest $R-R$ interval

Chronic
Persistent

Study group $(\mathrm{n}=280)$

72 (8.7)

162 (57.9)

$54.9(21-75.7)$

246 (87.8)

34 (13.2)

219 (78.2)

60 (21.4)

44 (15.7)

41 (14.6)

72 (25.7)

61 (21.8)

25 (8.9)

14 (5)

15 (5.6)

$60(50-65)$

68 (24.1)

45 (41-49)

16 (5.8)

22 (7.9)

$12(2-25)$

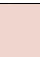

74

$74(67-84)$
$44(37-51)$
$142(115-162)$
$10(3.6)$
$22(7.9)$
$0(0-200)$
$115(41.1)$
$103(36.8)$
$137(48.9)$
$40(14.3)$

$75(68-84)$

73 (64-84)

0.59

$44(37-50)$

$43.5(37-54)$

0.89

$145(121-166)$

$133(106-152)$

0.01

$4(2)$

$6(7.7)$

0.02

$13(6.4)$

$9(11.5)$

0.15

$0(0-150)$

$10.5(0-400)$

61 (30.2)

$42(53.9)$

0.04

$107(53)$

30 (38.5)

R-R intervals $\geq 2 \mathrm{~s}, \mathrm{n}$, median (IQR)

$4(0-88.5)$

$34(16.8)$

$6(7.7)$

\section{Medication use at the time of 24 -hour ECG}

\begin{tabular}{ll}
$\beta$-Blockers & $243(86.8)$ \\
\hline$\beta$-Blockers equivalent dosages, mg, median (IQR) & $50(50-100)$ \\
\hline Verapamil & $9(3.2)$ \\
\hline Diltiazem & $2(0.7)$ \\
\hline Digoxin & $72(25.7)$ \\
\hline
\end{tabular}

$10(0-100)$

$0(0-35)$

$171(84.7)$
$100(50-100)$
$6(3)$
$1(0.5)$
$48(23.8)$

$72(92.3)$

0.02

0.001

Data are presented as number (percentage) of patients unless otherwise indicated.

Abbreviations: CABG, coronary artery bypass grafting; COPD, chronic obstructive pulmonary disease; ECG, electrocardiography; IQR, interquartile range; LBBB, left bundle branch block; LVEF, left ventricular ejection fraction; PCI, percutaneous coronary intervention; RBBB, right bundle branch block; VES, ventricular extrasystole 
TABLE 2 Death and survival rates in patients implanted with a pacemaker or defibrillator during follow-up according to the longest R-R interval on index 24-hour electrocardiography

\begin{tabular}{llllll} 
R-R interval, $\mathbf{s}$ & Survivors, $\mathbf{n}$ & $\begin{array}{l}\text { Survivors implanted with } \\
\text { pacemaker or defibrillator, } n(\%)\end{array}$ & $\begin{array}{l}\text { Deceased } \\
\text { patients, } n\end{array}$ & $\begin{array}{l}\text { Deceased patients implanted with } \\
\text { pacemaker or defibrillator, } n(\%)\end{array}$ & $\begin{array}{c}P \text { value } \\
0-1.9\end{array}$ \\
\hline $2-2.9$ & 61 & $5(8.2)$ & 42 & $1(2.4)$ & 0.42 \\
\hline $3-4.9$ & 107 & $3(2.8)$ & 30 & $3(10)$ & 0.23 \\
\hline$\geq 5$ & 33 & $7(21.1)$ & 6 & $3(50)$ & 0.44 \\
\hline
\end{tabular}

TABLE 3 Predictors of mortality in the proportional Cox regression model with (full model) and without significant 24-hour electrocardiographic parameters (adjusted stepwise regression)

\begin{tabular}{|c|c|c|c|c|c|}
\hline \multirow[t]{2}{*}{ Parameter } & \multicolumn{2}{|c|}{ Full model } & \multicolumn{2}{|c|}{ Adjusted stepwise regression } & \multirow{2}{*}{$\begin{array}{l}\text { Points in the risk score } \\
\text { system, } n\end{array}$} \\
\hline & $\mathrm{HR}(95 \% \mathrm{CI})$ & $P$ value & HR $(95 \%$ CI $)$ & $P$ value & \\
\hline Male sex & $1.65(0.98-2.79)$ & 0.06 & & & \\
\hline Age $\geq 70 y$ & $2.17(1.23-3.85)$ & 0.008 & $1.9(1.1-3.3)$ & 0.02 & 2 \\
\hline Renal failure grade $4-5$ & $8.4(3.43-20.5)$ & $<0.001$ & $8.8(4-9.1)$ & $<0.001$ & 8 \\
\hline Ischemic heart disease & $0.55(0.16-1.87)$ & 0.34 & & & \\
\hline $\mathrm{PCI} / \mathrm{CABG}$ & $4.36(1.49-12.7)$ & 0.007 & $2.7(1.6-4.5)$ & $<0.001$ & 3 \\
\hline COPD & $2.27(1.04-5)$ & 0.04 & $2.96(1.46-5)$ & 0.003 & 3 \\
\hline Myocardial infarction & $1.19(0.46-3.11)$ & 0.72 & & & \\
\hline Cancer & $1.7(0.7-4.14)$ & 0.24 & & & \\
\hline Diabetes & $0.5(0.19-1.34)$ & 0.17 & & & \\
\hline LVEF $<50 \%$ & $1.26(0.69-2.29)$ & 0.45 & & & \\
\hline $\begin{array}{l}\text { History } 0 \text { of pacemaker or } \\
\text { defibrillator implantation }\end{array}$ & $0.41(0.14-1.2)$ & 0.1 & & & \\
\hline Persistent atrial fibrillation & $0.5(0.18-1.33)$ & 0.17 & & & \\
\hline LBBB & $3.06(1.14-8.2)$ & 0.03 & $2.51(1.94-6.09)$ & 0.04 & 3 \\
\hline RBBB & $1.41(0.57-3.51)$ & 0.46 & & & \\
\hline Maximum heart rate $\geq 142$ bpm & $0.69(0.41-1.16)$ & 0.16 & & & \\
\hline $\begin{array}{l}\text { Pacemaker or defibrillator } \\
\text { implantation during follow-up }\end{array}$ & $0.76(0.31-1.82)$ & 0.53 & & & \\
\hline VESs $>5$ per 24 hours & $1.37(0.84-2.24)$ & 0.2 & & & \\
\hline The longest $\mathrm{R}$ - $\mathrm{R}$ interval $\geq 2 \mathrm{~s}$ & $0.31(0.18-0.52)$ & $<0.001$ & $0.38(0.24-0.62)$ & $<0.001$ & $\begin{array}{l}-3(1 / 0.38 \text { rounded }) \text { means } \\
\text { the presence of the factor } \\
\text { decreases the risk of death }\end{array}$ \\
\hline $\begin{array}{l}R-R \text { intervals } \geq 2 \text { s more than } 770 \text { per } \\
24 \text { hours, } n\end{array}$ & $4.6(1.2-17.6)$ & 0.03 & $5.98(2.1-17.34)$ & 0.01 & 6 \\
\hline
\end{tabular}

For each case, the score was calculated by adding the number of points for variables such as age, sex, PCI/CABG, LBBB, COPD, and renal failure grade 4 or 5 and compared using the receiver operating characteristic curves with the score calculated with the same variables and additionally variables derived from 24 -hour ECG: an interval lasting 2 seconds or longer, and the number of intervals exceeding 770 per 24 hours.

Abbreviations: HR, hazard ratio; others, see TABLE 1

the duration of the longest $\mathrm{R}-\mathrm{R}$ intervals and mortality as well as to assess the proportional hazards assumption, which was not violated. The proportional hazards Cox regression analysis was performed to identify factors related to long-term survival. The model was built on the basis of a comparison between deceased patients and survivors. The parameters that differed between the groups at a significance level of a $P$ value of less than 0.15 were included in the model. Moreover, data on pacemaker or defibrillator implantation after the index 24-hour ECG and during follow-up as well as on the presence of diabetes and COPD were included in the analysis.

The receiver operating characteristic (ROC) curves were constructed using the Youden 
index to determine the cutoff points for age, the number of VESs during 24-hour ECG, maximum heart rate, and the number of ventricular pauses to distinguish between deceased patients and survivors. These variables were dichotomized according to the cutoff points and included in the multivariable analysis.

Subsequently, a scoring system was developed based on the results of the multivariable model. A weight was assigned to each independent predictor of mortality by rounding to the nearest whole number, hazard ratio (HR) greater than 1, and for HR less than 1, the inverse of $H R$ was also rounded to the nearest whole number. The individual score was obtained by summarizing the weights of predictors with HR greater than 1 and subtracting the weights with HR less than 1 for each patient. Performance (area under the ROC curve [AUC], sensitivity, and specificity) was then calculated for the full model including 24-hour ECG parameters and compared with the model without these parameters using the Hanley algorithm. A $P$ value of less than 0.05 was considered significant.

RESULTS The baseline characteristics of the study group are presented in TABLE1. The mean (SD) age was 72 (8.7) years and there were 162 men $(57.9 \%)$. The median (IQR) follow-up was 4.5 (0.1-13.4) years. During the follow-up, there were 78 deaths after a median (IQR) time of 3.5 (1.6-5.4) years. Reduced LVEF was reported in about $25 \%$ of patients. Most patients received medications that potentially affected the heart rate (TABLE 1). $\beta$-Blockers were used by 243 (87\%) patients: bisoprolol 108 (38.5\%), metoprolol 83 (30\%), carvedilol 30 (11\%), nebivolol 18 (6\%), atenolol 1 (0.3\%), sotalol 5 ( $2 \%)$, betaxolol (3\%). The difference of equivalent doses of $\beta$-blockers between deceased patients and surviving patients was not statistically significant. Only $10.4 \%$ of patients did not take $\beta$-blockers, calcium channel blockers, or digoxin.

A comparison between patients who died and those who survived is presented in TABLE 1. Compared with survivors, deceased patients were older, more frequently had a history of ischemic heart disease, lower left ventricular ejection fraction, renal failure grade $4-5$, a greater number of VESs, and more frequently R-R interval less than 2 seconds during 24-hour ECG.

In univariate Cox regression analysis, patients with AF during the 24-hour ECG and no R-R intervals lasting 2 seconds or longer had a higher mortality rate than those with such intervals present (HR, 2.56; 95\% CI, 1.63-4.03; $P<0.001$ ).

Pacemaker or defibrillator implantation during follow-up Pacemaker or defibrillator implantation during follow-up was reported in 22 patients (8\%), of whom 7 (32\%) died and 15 (68\%) survived. Detailed outcomes are presented in TABLE 2. Pacemaker or defibrillator implantation had no impact on survival.

The ROC curve analysis revealed the following cutoff points for the prediction of mortality: for age, 70 years or older (AUC, 0.609; 95\% CI, $0.536-0.682 ; P=0.003)$; for the number of VESs, more than 5 (AUC, 0.579; 95\% CI, 0.5050-0.654; $P=0.037$ ); for the maximum heart rate, more than 142 bpm (AUC, 0.591; 95\% CI, 0.517-0.665; $P=0.02)$, and for the number of R-R intervals lasting 2 seconds or longer, more than 770 (AUC, 0.616 ; 95\% CI, 0.538-0.693; $P=0.004$ ). These parameters were included in the multivariable-adjusted Cox regression analysis.

As presented in TABLE 3, Cox regression analysis revealed the following parameters to be independent predictors of mortality: older age ( $\geq 70$ years), history of renal failure grade 4 or 5 , history of percutaneous coronary intervention or coronary artery bypass grafting (or both), COPD, LBBB, and a high number ( $>770)$ or the absence of R-R intervals lasting 2 seconds or longer. In FIGURE 1, Kaplan-Meier curves for survival according to the longest duration of the R-R interval less than 2 seconds and 2 seconds or more were presented.

The AUC for the model without the significant ECG predictors of mortality (a high number or the absence of R-R intervals $\geq 2 \mathrm{~s}$ ) was 0.688 (95\% CI, 0.618-0.758), and improved to 0.748 (95\% CI, 0.686-0.81; $P=0.02$ ) after inclusion of the ECG predictors. In FIGURE2, receiver operating characteristic curves of the model with and without significant 24-hour electrocardiographic parameters were presented.

DISCUSSION The main finding of our study is that either a very high number or total absence of ventricular pauses lasting longer than 2 seconds during AF predicts long-term mortality.

The ventricular response during AF is typically characterized by irregular R-R intervals. ${ }^{16,17}$ The duration of $\mathrm{R}-\mathrm{R}$ intervals during AF depends on intrinsic and extrinsic factors affecting the atrioventricular (AV) node. The intrinsic factors include concealed conduction, refractoriness, and excitability of the AV node, a higher rate of atrial impulses through the node, variations in autonomic nervous system tone, use of drugs, and the presence or absence of AV nodal disease. As R-R intervals are controlled by the activity of the autonomic system, the parasympathetic branch of which is more active at night, the pauses are more prevalent at night than during the day. ${ }^{18}$ In a study by Sairaku et al, ${ }^{19}$ atrioventricular conduction parameters assessed after AF ablation did not differ between patients with no pauses, moderate pauses $(<3 \mathrm{~s})$, and those with longer pauses during AF. However, a disease of the AV node 


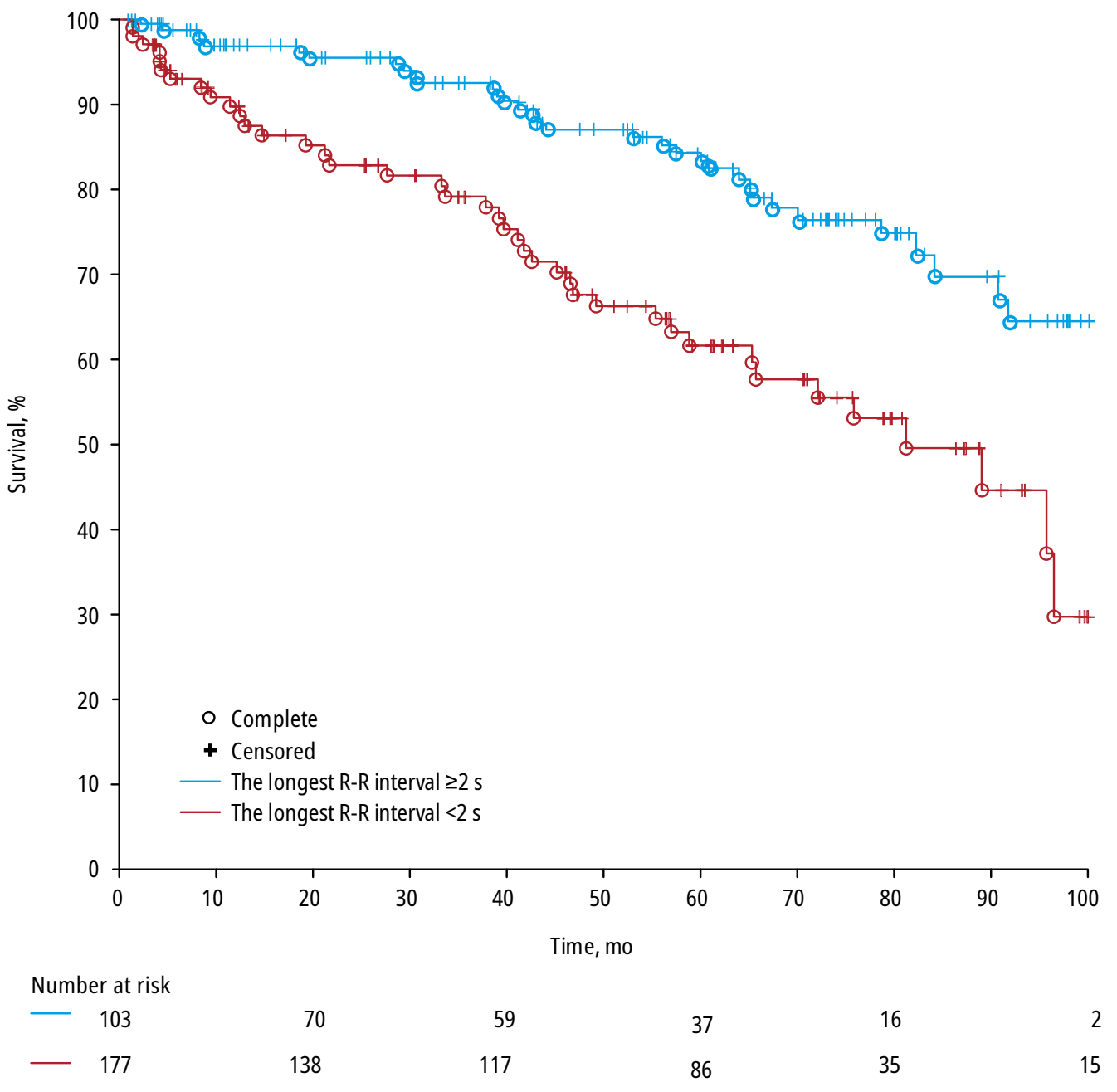

FIGURE 1 Kaplan-Meier curves for survival according to the longest duration of the R-R interval

or the His-Purkinje system may result in third-degree AV block during AF with slow ventricular rate. ${ }^{20}$ There is no clear definition of a too long R-R interval in AF. Neither European nor American guidelines define any threshold for the duration or number of pauses as an indication for pacemaker implantation. ${ }^{20,21}$

Different risk scores have been developed to predict mortality in $\mathrm{AF}$, such as the $\mathrm{ABC}$ risk score, which includes age, biomarkers, and clinical history of heart failure but no markers of cardiovascular autonomic dysfunction or ECG-based parameters. ${ }^{22}$ Hayano et $\mathrm{al}^{18}$ reported that circadian rhythm of $\mathrm{AV}$ conduction predicted long-term survival in patients with chronic AF; however, they used parameters that are not easily available in daily clinical practice. In the present study, we found a strong association between the presence of a moderate number of ventricular pauses and better survival.

There are several biologically plausible mechanisms that may explain the association between longer ventricular pauses and survival. First, inadequate rate control may directly affect cardiac health, although this hypothesis was not supported but the results of the RACE II (the Rate Control Efficacy in Permanent Atrial Fibrillation II) study, in which lenient and strict rate control strategies were associated with a similar risk of death. ${ }^{23}$ However, in the RACE II study, mainly resting ECG recordings were used to determine rate control, while our study clearly suggested that there may be other parameters of heart rate in patients with AF that may have a closer relation to mortality. Second, the absence of ventricular pauses may be a marker of cardiac autonomic dysfunction and a higher hyperadrenergic drive, which might be causally related to mortality. Finally, a high minimum heart rate and absence of ventricular pauses may indicate poor general health, without any causal relationship to mortality.

The sympathetic nervous system innervates the interventricular groove of the heart ${ }^{24}$; therefore, an increased sympathetic drive, regardless of the cause, can result in an elevated minimum heart rate and the absence of ventricular pauses through sympathetic innervation of the AV node. This could have a direct effect on cardiac morbidity and mortality, because it has been 


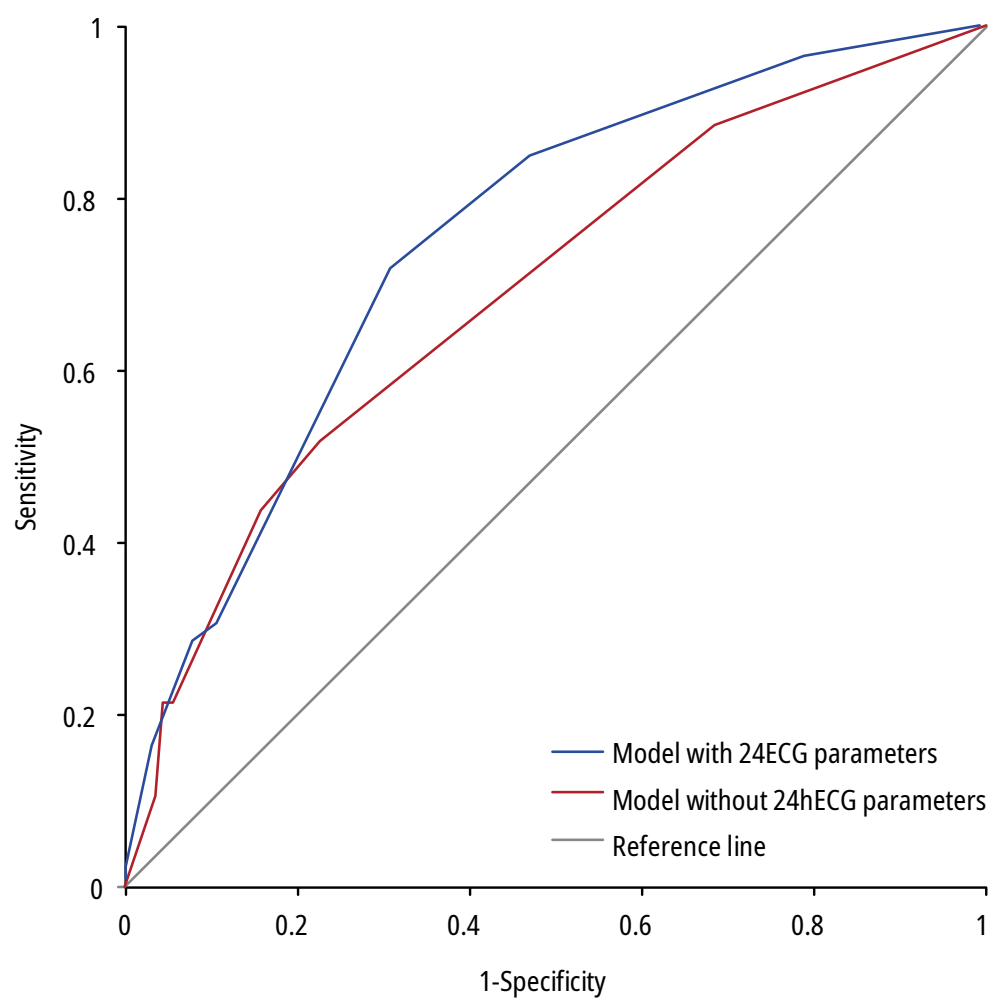

FIGURE 2 Receiver operating characteristic curve of the model with and without significant 24-hour electrocardiographic (ECG) parameters

proposed that a high resting heart rate is linked to atherosclerosis and ischemic heart disease as well as to reduced LVEF. ${ }^{25}$ This hypothesis may be also supported by the reports on the association between increased resting heart rate and mortality both in the general population ${ }^{14,25,26}$ and in patients with heart failure. ${ }^{27}$

Apart from contributing to cardiac autonomic dysfunction, a high sympathetic drive could also indicate poor general health status. Therefore, the association between 24-hour ECG parameters related to sympathetic drive and mortality could be due to poorer general health manifesting as a higher minimum heart rate and shorter ventricular pauses.

In recent years, there has been growing interest in therapies aiming at cardiac autonomic modulation, such as cardiac sympathetic denervation, ablation, or modulation of the ganglionated plexi, carotid body stimulation, and spinal cord stimulation. However, the results reported thus far have been conflicting, ${ }^{24}$ except for ganglionated plexi ablation, which shows some promise in AF control. ${ }^{28}$ Our study raises the question of whether 24-hour ECG parameters might be useful in identifying a subgroup of AF patients who might benefit from these interventions.

Another significant finding of our study is that a moderate number of ventricular pauses may not be an indication for pacing. On the other hand, a high number of ventricular pauses is related to increased mortality; therefore, it constitutes a more important indication for pacemaker implantation than pause duration. It is possible that a high minimum heart rate and the absence of ventricular pauses may be related to heart failure with preserved ejection fraction, because reduced cardiac functional reserve may induce compensatory mechanisms including a higher heart rate.

The importance of activity of the parasympathetic nervous system may be different in different populations. In almost 20-year younger population of patients with lower incidence of comorbidities than in our group, the parasympathetic nervous system promoted recurrence of AF after pulmonary vein isolation. ${ }^{29,30}$

This study has several limitations that should be considered when interpreting the results. First, this was a single-center study including a small population of patients recruited over a long period of time and thus cannot be seen as a representative for the general population. Second, 24-hour ECG measurements were obtained in the routine clinical practice setting; therefore, some of the parameters may be subject to a measurement error, particularly the frequency of VESs, which can be difficult to distinguish from beats with aberrant conduction. Also we could not exclude errors in pause duration measurement and pause counting Moreover, narrow complex ventricular beats may have been missed. These possible measurement errors may have biased the results towards null for the association between VESs and mortality. Other 24-hour ECG parameters, such as heart rate variables and the presence of ventricular pauses, can be assumed to be more robust, because they are mainly derived directly from the ECG analysis software and are therefore less prone to human interpretation error. Finally, some clinical characteristics of the participants were lacking, such as the duration and changes of medical therapy and severity of comorbidities such as COPD and hypertension.

In conclusion, 24-hour ECG parameters may predict mortality in patients with permanent or persistent AF. Presence of maximal ventricular intervals lasting more than 2 seconds during atrial fibrillation may indicate better survival, while a very high number of such ventricular pauses represents a risk factor for death.

\section{ARTICLE INFORMATION}

\section{CONFLICT OF INTEREST None declared.}

OPEN ACCESS This is an Open Access article distributed under the terms of the Creative Commons Attribution-NonCommercial-NoDerivatives 4.0 International License (CC BY-NC-ND 4.0), allowing third parties to download articles and share them with others, provided the original work is properly cited, not changed in any way, distributed under the same license, and used for noncommercial purposes only. For commercial use, please contact the journal office at kardiologiapolska@ptkardio.pl.

HOW TO CITE Zyśko D, Persson A, Obremska M, et al. The importance of the longest R-R interval on 24-hour electrocardiography for mortality prediction in patients with atrial fibrillation. Kardiol Pol. 2021; 79: 311-318. doi:10.33963/ KP.15820

\section{REFERENCES}

1 Benjamin EJ, Wolf PA, D'Agostino RB, et al. Impact of atrial fibrillation on the risk of death: the Framingham Heart Study. Circulation. 1998; 98: 946-952. 
2 Panchal G, Mahmood M, Lip GYH. Revisiting the risks of incident atrial fibrillation: a narrative review. Part 1. Kardiol Pol. 2019; 77: 430-436.

3 Panchal G, Mahmood M, Lip GYH. Revisiting the risks of incident atrial fibrillation: a narrative review. Part 2. Kardiol Pol. 2019; 77: 515-524.

4 Gómez-Outes A, Lagunar-Ruíz J, Terleira-Fernández AI, et al. Causes of death in anticoagulated patients with atrial fibrillation. J Am Coll Cardiol. 2016; 68: 2508-2521.

5 Johnson LS, Berntsson J, Juhlin T, et al. ST segment depression on 24-hour electrocardiography predicts incident atrial fibrillation in two population-based cohorts. Europace. 2018; 20: 429-434.

6 Johnson LS, Juhlin T, Juul-Möller $S$, et al. A prospective study of supraventricular activity and incidence of atrial fibrillation. Heart Rhythm. 2015; 12: 1898-1904.

7 Binici Z, Intzilakis T, Nielsen OW, et al. Excessive supraventricular ectopic activity and increased risk of atrial fibrillation and stroke. Circulation. 2010; 121: 1904-1911.

8 Persson AP, Fedorowski A, Hedblad B, et al. Heart rate and premature atrial contractions at $24 \mathrm{hECG}$ independently predict atrial fibrillation in a population-based study. Heart. 2020; 106: 287-291.

9 Hedblad B, Juul-Möller S, Svensson K, et al. Increased mortality in men with ST segment depression during $24 \mathrm{~h}$ ambulatory long-term ECG recording. Results from prospective population study 'Men born in 1914', from Malmö, Sweden. Eur Heart J. 1989; 10: 149-158.

10 Van Gelder IC, Rienstra M, Crijns HJ, Olshansky B. Rate control in atrial fibrillation. Lancet. 2016; 388: 818-828.

11 Spallone V, Ziegler D, Freeman R, et al. Cardiovascular autonomic neuropathy in diabetes: clinical impact, assessment, diagnosis, and management. Diabetes Metab Res Rev. 2011; 27: 639-653.

12 Fedorowski A, Hedblad B, Engstrom G, et al. Orthostatic hypotension and long-term incidence of atrial fibrillation: the Malmö Preventive Project. J Intern Med. 2010; 268: 383-389.

13 Fedorowski A, Stavenow L, Hedblad B, et al. Orthostatic hypotension predicts all-cause mortality and coronary events in middle-aged individuals (The Malmo Preventive Project). Eur Heart J. 2010; 31: 85-91.

14 Jensen MT, Suadicani P, Hein HO, Gyntelberg F. Elevated resting heart rate, physical fitness and all-cause mortality: a 16-year follow-up in the Copenhagen Male Study. Heart. 2013; 99: 882-887.

15 Di Lenarda A, Remme W], Charlesworth A, et al. Exchange of beta-blockers in heart failure patients. Experiences from the poststudy phase of COMET (the Carvedilol or Metoprolol European Trial). Eur J Heart Fail. 2005; 7: 640-649.

16 Black N, D'Souza A, Wang Y, et al. Circadian rhythm of cardiac electrophysiology, arrhythmogenesis, and the underlying mechanisms. Heart Rhythm. 2019; 16: 298-307.

17 Raeder EA. Circadian fluctuations in ventricular response to atrial fibrillation. Am J Cardiol. 1990; 66: 1013-1016.

18 Hayano J, Ishihara S, Fukuta $\mathrm{H}$, et al. Circadian rhythm of atrioventricular conduction predicts long-term survival in patients with chronic atrial fibrillation. Chronobiol Int. 2002; 19: 633-648.

19 Sairaku A, Nakano Y, Oda N, et al. Atrioventricular conduction properties in patients with prolonged pauses undergoing ablation of longstanding persistent atrial fibrillation: do pauses during atrial fibrillation matter? J Interv Card Electrophysiol. 2012; 34: 277-285.

20 European Society of Cardiology (ESC); Brignole M, Auricchio A, Baron-Esquivias G, et al. 2013 ESC guidelines on cardiac pacing and cardiac resynchronization therapy: the task force on cardiac pacing and resynchronization therapy of the European Society of Cardiology (ESC). Developed in collaboration with the European Heart Rhythm Association (EHRA). Europace. 2013; 15: 1070-1118.

21 Kusumoto FM, Schoenfeld MH, Barrett C, et al. Guideline on the evaluation and management of patients with bradycardia and cardiac conduction delay: a report of the American College of Cardiology/American Heart Association Task Force on Clinical Practice Guidelines and the Heart Rhythm Society. Circulation. 2019; 140: e382-e482.

22 Hijazi Z, Oldgren J, Lindback J, et al. A biomarker-based risk score to predict death in patients with atrial fibrillation: the $A B C$ (age, biomarkers, clinical history) death risk score. Eur Heart J. 2018; 39: 477-485.

23 Van Gelder IC, Groenveld HF, Crijns HJ, et al. Lenient versus strict rate control in patients with atrial fibrillation. N Engl J Med. 2010; 362: 1363-1373.

24 Herring N, Kalla M, Paterson D. The autonomic nervous system and cardiac arrhythmias: current concepts and emerging therapies. Nat Rev Cardiol. 2019; 16: $707-726$

25 Fox K, Borer JS, Camm AJ, et al. Resting heart rate in cardiovascular disease. J Am Coll Cardiol. 2007; 50: 823-830.

26 Aune D, Sen A, ó'Hartaigh B, et al. Resting heart rate and the risk of cardiovascular disease, total cancer, and all-cause mortality - a systematic review and dose-response meta-analysis of prospective studies. Nutr Metab Cardiovasc Dis. 2017; 27: 504-517.

27 Komajda M. Heart rate in chronic heart failure: an overlooked risk factor. Eur Heart J. 2015; 36: 648-649.

28 Pokushalov E, Romanov A, Artyomenko S, et al. Ganglionated plexi ablation for longstanding persistent atrial fibrillation. Europace. 2010; 12: 342-346.
29 Marinković M, Mujović N, Vučićević $V$, et al. A square root pattern of changes in heart rate variability during the first year after circumferential pulmonary vein isolation for paroxysmal atrial fibrillation and their relation with long term arrhythmia recurrence. Kardiol Pol. 2020; 78: 209-218.

30 Shantsila A, Gupta D, Lip GYH. Heart rate variability changes following catheter ablation for atrial fibrillation. Kardiol Pol. 2020; 78: 179-180. 